\title{
Students' conceptual performance on synthesis physics problems with varying mathematical complexity
}

\author{
Bashirah Ibrahim, ${ }^{1}$ Lin Ding, ${ }^{1, *}$ Andrew F. Heckler, ${ }^{2}$ Daniel R. White, ${ }^{2}$ and Ryan Badeau ${ }^{2}$ \\ ${ }^{1}$ Department of Teaching and Learning, The Ohio State University, Columbus, Ohio 43210, USA \\ ${ }^{2}$ Department of Physics, The Ohio State University, Columbus, Ohio 43210, USA
}

(Received 22 December 2016; published 28 June 2017)

\begin{abstract}
A body of research on physics problem solving has focused on single-concept problems. In this study we use "synthesis problems" that involve multiple concepts typically taught in different chapters. We use two types of synthesis problems, sequential and simultaneous synthesis tasks. Sequential problems require a consecutive application of fundamental principles, and simultaneous problems require a concurrent application of pertinent concepts. We explore students' conceptual performance when they solve quantitative synthesis problems with varying mathematical complexity. Conceptual performance refers to the identification, follow-up, and correct application of the pertinent concepts. Mathematical complexity is determined by the type and the number of equations to be manipulated concurrently due to the number of unknowns in each equation. Data were collected from written tasks and individual interviews administered to physics major students $(N=179)$ enrolled in a second year mechanics course. The results indicate that mathematical complexity does not impact students' conceptual performance on the sequential tasks. In contrast, for the simultaneous problems, mathematical complexity negatively influences the students' conceptual performance. This difference may be explained by the students' familiarity with and confidence in particular concepts coupled with cognitive load associated with manipulating complex quantitative equations. Another explanation pertains to the type of synthesis problems, either sequential or simultaneous task. The students split the situation presented in the sequential synthesis tasks into segments but treated the situation in the simultaneous synthesis tasks as a single event.
\end{abstract}

DOI: 10.1103/PhysRevPhysEducRes.13.010133

\section{INTRODUCTION}

Problem solving has played a central role in physics education [1]. However, the vast majority of the problems employed are relatively simplified compared to real-world problems that students will eventually encounter. For example, assigned problems are typically structured to elicit the application of only a single major physics concept, i.e., the one being taught at the time [2]. Employing only singleconcept problems deprives students of the opportunity to have in-depth engagement in the process of identifying the relevant concepts from a range of topics, and reflecting on proper approaches to handle a more complex problem involving the application of multiple concepts.

Over the last few decades there have been significant physics education research efforts in the domain of problem solving $[3,4]$. However, as if to mirror the instructional

\footnotetext{
*Corresponding author. ding.65@osu.edu

Published by the American Physical Society under the terms of the Creative Commons Attribution 4.0 International license. Further distribution of this work must maintain attribution to the author(s) and the published article's title, journal citation, and DOI.
}

practices in typical classrooms, most previous studies have utilized only single-concept problems [1,5-11].

\section{A. Synthesis physics problems}

In contrast to previous efforts, in this study we investigate students' handling of synthesis problems, which are tasks consisting of two or more distinct concepts, typically from different chapters and separated in the teaching timeline [2]. The core of a synthesis problem is integration of multiple concepts as well as the mathematical formulations emerging from the application of the concepts in order to build a solution. A search for literature on synthesis problem solving in the domain of physics so far indicates a paucity of studies which explicitly address this research area. Ogilvie [12] used what is termed as multifaceted context-rich problems to create epistemic doubts among introductory physics students in order to shift their beliefs about problem solving approaches from a novice to an expertlike one. In a follow-up study, Antonenko et al. [13] utilized an online problem-based learning environment to identify the information used by students when solving multifaceted problems. They reported that with practice the students tended to qualitatively analyze the problems at the beginning of the problem solving activity and focus more on conceptual information. The physics education research group at the 


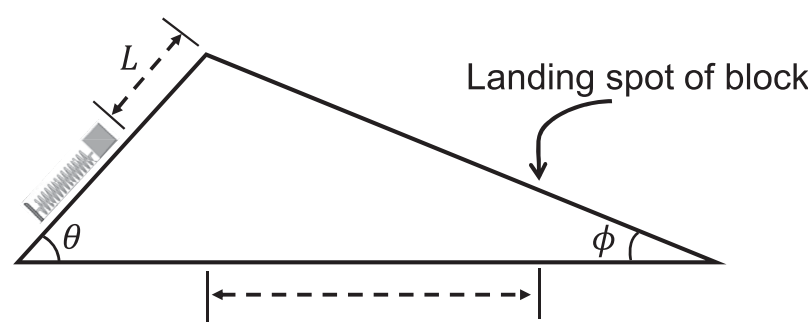

$R$

An apparatus uses a spring launcher to fire a block (mass $2 \mathrm{~kg}$ ) over a ridge. The spring (spring constant, $\mathrm{k}=3000 \mathrm{~N} / \mathrm{m}$ ) is compressed $0.1 \mathrm{~m}$ and releases the block from its equilibrium position, which is located a distance $L=0.5 \mathrm{~m}$ from the crest. If the angles shown are $\theta=35^{\circ}$ and $\phi=25^{\circ}$, what is the horizontal distance $R$ from the crest to where the block hits the slope on the other side?

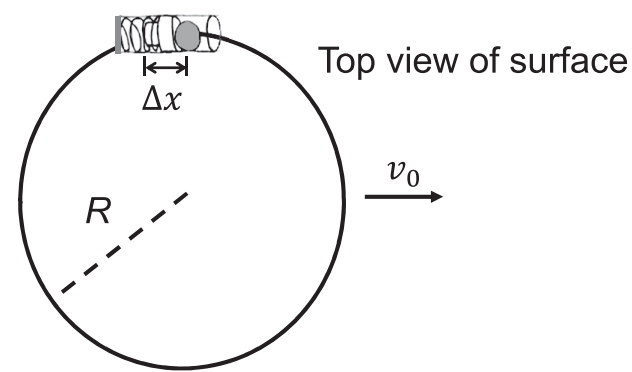

A disc of mass $3.0 \mathrm{~kg}$ and radius $R=0.75 \mathrm{~m}$ is sliding (face down) at velocity $v_{o}$ along a frictionless surface. A spring launcher is fixed outside the edge of the disc. The spring (spring constant, $k=49.0 \mathrm{kN} / \mathrm{m}$ ) is compressed a distance $\Delta x$ and releases a projectile of mass $6.0 \mathrm{~kg}$ from its equilibrium position. After release, the velocity of the projectile relative to the surface is $v_{p}=11.0 \mathrm{~m} / \mathrm{s}$. Assume that the mass of the launcher is small and that the spring releases the projectile very rapidly. If the initial compression of the spring was $\Delta x=17.5 \mathrm{~cm}$, what was the initial velocity of the disc, $v_{o}$ ?

FIG. 1. Types of synthesis problems: (a) sequential and (b) simultaneous.

University of Minnesota used problems combining different concepts in their design of web-based computer coaches to develop expertlike problem solving approaches among introductory physics students [14]. Further, Lin and Singh [15] utilized problems comprising multiple concepts in their study investigating students' ability to solve isomorphic problems using analogical reasoning.

Although there may be a number of ways to merge concepts in synthesis problems, in our study, we focus on two common synthesis problem types, namely, sequential and simultaneous synthesis problems. An example of each type of synthesis problem is shown in Fig. 1.

Figure 1(a) is a sequential synthesis problem. The situation can be analyzed as two consecutive events: a block is first propelled from a spring and then undergoes a trajectory to land on an inclined surface leading to the consecutive application of two pertinent concepts. Even though the two events are parts of one complex event and are connected by several variables in the problem, it is possible (and physically correct) to consider one event at a time.

Figure 1(b) is a simultaneous synthesis problem. The situation must be analyzed as events occurring simultaneously: a disc sliding on a frictionless surface while the spring releases a projectile causing the motion of the system to spin. In this situation, the events of energy transfer and a change in the system's rotational and translational motion occur simultaneously, necessarily leading to the concurrent application of the fundamental concepts. In other words, for sequential synthesis problems, multiple concepts (typically involving multiple physical events) must be applied at different points in time, and for simultaneous synthesis problems the multiple concepts must be applied at the same point in time.

Initial studies on quantitative synthesis problem solving by some of the authors of this study $[2,16,17]$ have highlighted the key role of guided conceptual scaffolding promoting an expert approach to problem solving among students. However, these studies have also reported that despite conceptual scaffolding, most students were unsuccessful in determining the final correct answer. Further investigation [18] has led us to propose three necessary components (or bottlenecks) to synthesis problems, namely, single-concept proficiency, concept identification, and application. Consider a synthesis problem that involves concept $\mathrm{A}$ and $\mathrm{B}$. The solver must (i) be adequately proficient at solving the problems involving A only and B only, (ii) recognize that the problem necessarily involves both concepts $\mathrm{A}$ and $\mathrm{B}$, and (iii) be proficient at applying both concepts in concert to solve the problem. White et al. [18] has investigated and documented the first two bottlenecks, single-concept proficiency and concept identification.

In this study, we examine the role of a potentially important factor in the three bottlenecks, namely, the mathematical complexity of the problem.

\section{B. Mathematical complexity}

We are interested in characterizing how mathematical complexity influences the synthesis problem-solving process. One might expect that mathematical complexity could influence all three components (bottlenecks) of synthesis problem solving. We are especially interested in how it 
affects the second and third components, namely, the identification and the application of the multiple concepts in synthesis problem solving.

The mathematical complexity of a solution may depend on several factors, including the number and the type of concepts relevant to the posed problem and the mathematical structure in which the to-be-determined variables are imbedded in the posed problem. For example, in mathematics, a single-unknown problem posed as a "singlereference" problem [e.g., $(x-1) / 3=4$ ] is less complex (and less difficult) than a problem posed as a "double reference" problem [e.g., $x-0.3 x=7$ ] [19]. Furthermore, for multistep problems, there is another factor of complexity called the composition effect, first documented by Heffernan and Koedinger [20]. Specifically, they found that student performance on "composed" problems involving two unknowns was significantly worse than the combined performance on two match problems with one unknown each. This is similar to our findings of synthesis versus single-concept problems [18].

Therefore, consistent with previous research, we make the following hypotheses. First, we propose that a problem with $n$ unknowns represented simultaneously in a system of $n$ equations is mathematically more complex than a problem with $n$ unknowns represented individually in $n$ separate equations. Second, we propose that a problem involving unknowns in nonlinear equations (such as quadratic and trigonometric) is more mathematically complex than a problem having unknowns in linear equations. Thus, we operationally define mathematical complexity as the number and the type of equations to be solved simultaneously due to the number of unknowns in each equation. In this study, the physics itself does not change per se, but rather the variables (knowns and unknowns) are manipulated such that the mathematical complexity is varied.

\section{Mathematical complexity and its influence on student conceptual performance}

It is well documented that students tend to use the plugand-chug approach to tackle single-concept problems by structuring solution paths based on variables to be determined [21-25]. Often, students view equations as mere computational tools and struggle to attribute physics meaning to the mathematical symbols [26-29]. Although they are fluent in manipulating simple equations with one unknown [30], their focus is mainly on determining a quantitative solution [31-33]. Thus, many studies have advocated the importance of including conceptual reasoning in the problem solving process, particularly when manipulating equations to find a solution [29,30,32-35]. Further, the multistep problem solving strategies designed to improve students' performance typically emphasize conceptual reasoning, such as formulating equations and evaluating the final answers [4,26,36-38]. More recently, Kuo et al. [29] studied how students used formulated equations for problem solving and argued that the blending of conceptual reasoning and symbolic manipulations was an essential problem solving skill.

The studies mentioned above highlight the key role of conceptual reasoning when solving quantitative singleconcept problems. In our study, we use quantitative synthesis problems with varying mathematical complexity. We emphasize students' conceptual reasoning by tapping into their performance on the identification, follow-up, and application of pertinent concepts underlying the synthesis problems to formulate the equations. In short, we explore the students' conceptual performance when tackling quantitative synthesis problems with varying mathematical complexity.

This study is important for at least two reasons. First, it emphasizes synthesis problems that cannot be solved simply by using plug and chug. Here, we uniquely focus on students' conceptual reasoning in situations involving multiple physics topics. This line of work can provide opportunities for developing expertlike skills among our students by guiding their attention to underlying fundamentals. Second, the current study zooms in on mathematical complexity as a possible barrier in quantitative synthesis tasks. Our earlier work [18] has shown that students face a great challenge in identifying and applying multiple concepts in solving synthesis problems. This challenge can be attributed to multiple factors, one of which is mathematics. It is imperative that we understand the role of mathematical complexity, and particularly its influences on concept identification and application, in synthesis problem solving.

We posit that in solving quantitative synthesis problems, mathematical complexity may interfere with the students' conceptual performance. In this study, conceptual performance refers to the above-mentioned bottlenecks: identification of, follow-up on, and application of appropriate concepts. Mathematical complexity represents a potential difficultly for quantitative synthesis problem solving in the following ways. As the task's mathematical complexity increases, there is an increase in the number of unknowns in each equation. This in turn may result in not only an increase in the number of steps needed to solve for each unknown, but increased complexity of the solution process. For example, quadratic or trigonometric equations can emerge. This increased complexity may negatively impact the way students choose to tackle the physics principles underlying the problems, especially when they do not have a robust conceptual understanding. They may modify their formulated equations to a simpler form, omitting certain variables or crucial elements, hence incorrectly applying the concepts or disregarding certain principles. In short, we propose that mathematical complexity may negatively influence the students' decision on the relevance of particular concepts, their commitment to proceed, and their application of the chosen concepts.

We therefore address the following research question: How does mathematical complexity influence students' conceptual 
Simple: An apparatus uses a spring launcher to fire a block (mass $2 \mathrm{~kg}$ ) over a ridge. The spring (spring constant, $k=3000 \mathrm{~N} / \mathrm{m}$ ) is compressed $0.1 \mathrm{~m}$ and releases the block from its equilibrium position, which is located a distance $L=0.5 \mathrm{~m}$ from the crest. If the angles shown are $\theta=35^{\circ}$ and $\phi=25^{\circ}$, what is the horizontal distance $R$ from the crest to where the block hits the slope on the other side?

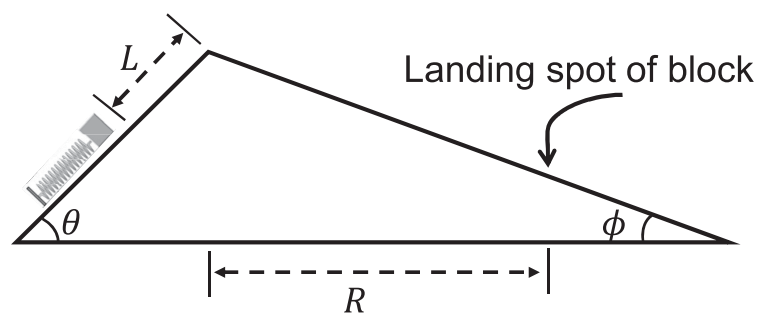

Intermediate: An apparatus uses a spring launcher to fire a block (mass $2 \mathrm{~kg}$ ) over a ridge. The spring (spring constant, $\mathrm{k}=3000 \mathrm{~N} / \mathrm{m}$ ) is compressed an unknown distance $x$, and releases the block from its equilibrium position, which is located a distance $L=0.5 \mathrm{~m}$ from the crest. The block lands on the opposite slope, a horizontal distance $R=1.3 \mathrm{~m}$ from the crest. If the angles shown are $\theta=35^{\circ}$ and $\phi=25^{\circ}$, what was the initial compression of the spring, $x$ ?

Complex: An apparatus uses a spring launcher to fire a block (mass $2 \mathrm{~kg}$ ) over a ridge. The spring (spring constant, $\mathrm{k}=3000 \mathrm{~N} / \mathrm{m}$ ) is compressed $0.1 \mathrm{~m}$ and releases the block from its equilibrium position, which is located a distance $L=0.5 \mathrm{~m}$ from the crest. The block lands on the opposite side, a horizontal distance $R=1.3 \mathrm{~m}$ from the crest. If $\phi=25^{\circ}$, what was the angle of the initial slope, $\theta$ ?
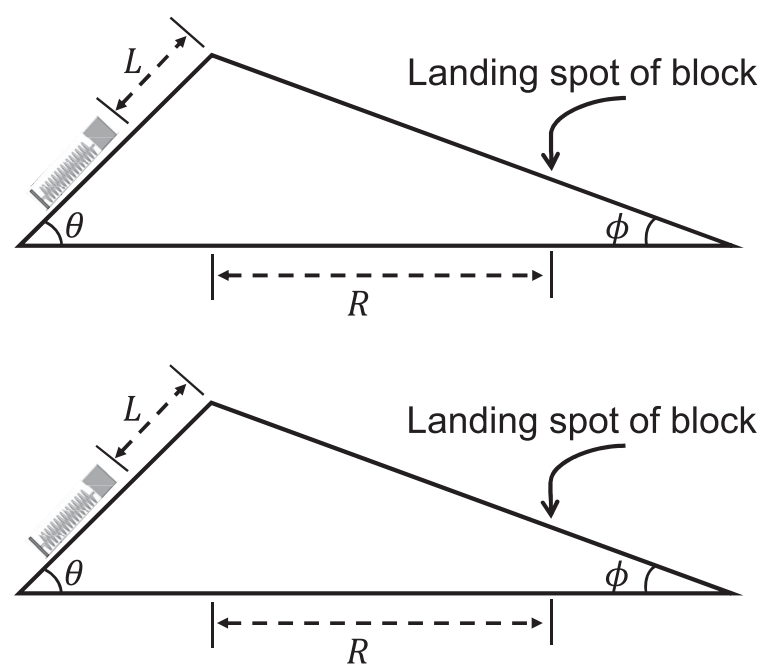

FIG. 2. The three versions of the sequential synthesis problems.

performance when they solve quantitative sequential and simultaneous synthesis physics problems? To answer this question, we compare the impact of mathematical complexity on students' abilities to identify the appropriate concepts, commitment to use them, and to correctly apply them when they handle sequential and simultaneous synthesis problems. We also explore possible reasons for the similarities or differences in student conceptual performance on the sequential and simultaneous synthesis tasks.

Two studies were conducted to gain insights into the different aspects of interest described above. Study 1 explores the effects of mathematical complexity on students' conceptual performance on sequential synthesis problems. Study 2 focuses on simultaneous synthesis tasks. Sections II and III present the methodology and outcomes of the two studies. The conclusions and discussions are highlighted in Sec. IV.

\section{STUDY 1: EFFECTS OF MATHEMATICAL COMPLEXITY ON STUDENT CONCEPTUAL PERFORMANCE IN SEQUENTIAL SYNTHESIS PROBLEMS}

Study 1 investigates students' conceptual performance on tackling a sequential synthesis problem with varying mathematical complexity. Sequential synthesis problems (as shown in Fig. 2) include a chronological occurrence of different events and hence require consecutive applications of multiple fundamental concepts.

\section{A. Method \\ 1. Sample}

The sequential synthesis problems were administered during the Fall of 2014. A cohort of 105 students, enrolled in a second year mechanics course for physics majors, participated in the study. Of these 105 students, 92 signed up to complete the written tasks only. The remaining 13 students participated in individual interviews. Our assignment of students to the written task or individual interviews was random. The students completed the sequential synthesis problems on energy conservation and projectile motion after being taught these concepts in lectures. They had opportunities to tackle multiconcept problems in class and as homework assignments. The course was traditionally taught and included a recitation where students worked in groups of 3-4.

\section{Sequential synthesis problems}

The sequential problems used in the study are presented in Fig. 2. The final set of problems was vetted with a group of physicists, physics education researchers, and students. We specifically checked that the students did not misinterpret the problem statements. We also ensured that the quantitative information presented is realistic, such that the final quantitative solution is rational, and the problems are based on concepts which have been taught in class. 
Energy conservation: $\frac{1}{2} \mathrm{k} x^{2}=\frac{1}{2} \mathrm{~m} v^{2}+\mathrm{mg}(L+x) \sin \theta \ldots .(1)$

\section{Projectile motion:}

$$
\begin{aligned}
& R=v \cos \theta t \ldots \ldots \ldots \ldots \\
& y=v \sin \theta t-\frac{1}{2} g t^{2} \\
& \tan \phi=\frac{-y}{R} \ldots \ldots \ldots \ldots \ldots \ldots
\end{aligned}
$$

FIG. 3. The four essential equations for solving the sequential synthesis problems.

Three versions of the same task were designed with varying mathematical complexity, which we call simple, intermediate, and complex. They are all based on the same situation of a block initially propelled from a spring on a plane inclined at an angle $\theta$. The block then undergoes a trajectory and lands on another ramp angled at $\phi$. Energy conservation and projectile motion are the two major concepts needed to solve the three versions of the problem.

In all three cases, four equations are needed to solve the problem. They are presented in Fig. 3.

Application of energy conservation to the given situation results in the formulation of Eq. (1) which identifies and relates different types of energy (spring potential energy, kinetic energy, and gravitational potential energy). Application of projectile motion gives rise to three equations: Eq. (2) and Eq. (3) for the horizontal and vertical motion, respectively, and Eq. (4), a trigonometry relating the angle $\phi$, the magnitude and the direction of the horizontal and vertical displacements. Even though these four fundamental equations must be handled concurrently, the situation consists of two distinct events, which occur sequentially.

Information about the known and unknown variables and the types of equations to be manipulated for the three versions of the sequential synthesis task is shown in Table I. As the task's mathematical complexity increases (from simple to complex task), the frequency of the unknowns appearing in the equations increases and the types of equations to be manipulated vary from being linear to quadratic with trigonometry embedded.

\section{Administration of sequential synthesis problems}

We collected data during the "flexible homework" assignment [39], which is a 1 hour session for students to engage in activities such as interviews and tutorials as a replacement for one of their homework assignments. The students would receive full credit for participating in the flexible homework. The sample comprises 105 students. 13 of the 105 students signed up for a 1 hour individual interview session. Each student was randomly assigned to one version of the problem: simple $(n=4)$, intermediate $(n=5)$, and complex $(n=4)$. In each session, the student was initially allocated 30 minutes to solve the problem. The interview immediately followed lasting a maximum of approximately 30 minutes.

The remaining 92 students signed up to complete the written tasks only. They were randomly assigned the simple $(n=30)$, intermediate $(n=31)$, and complex $(n=31)$ version of the problem. There was no significant difference in the average course grade across the three groups $[F(2,91)=0.127, p=0.881]$ indicating that the three samples were at the same level. Each student completed the assigned problem individually, without consulting their peers. Regardless of the task's mathematical complexity, all the students took approximately 30 minutes to solve the problems.

\section{Analysis of sequential synthesis problems}

Written tasks.-From the written tasks, the students' responses were analyzed in three levels at the order of

TABLE I. Known and unknown variables and types of equations across sequential synthesis tasks with increasing mathematical complexity ( $m$ : mass of block, $k$ : spring constant, $L$ : equilibrium position, $\phi$ : angle of final slope, $x$ : initial compression of spring, $\theta$ : angle of initial slope, $R$ : horizontal distance, $v$ : block's velocity at crest of the ramp, $y$ : block's final vertical position, $t$ : time taken by block to hit the landing spot).

\begin{tabular}{lccc}
\hline \hline & Simple task & Intermediate task & Complex task \\
\hline Common given information & $x$ and $\theta$ & $m, k, L$, and $\phi$ & $R$ and $x$ \\
Additional given information & $R$ & $R$ and $\theta$ & $\theta$ \\
Variable to solve for & & $x$ & $2:(v, \theta)$ \\
Number of unknowns in each equation & $1:(v)$ & $2:(v, x)$ & $3:(v, \theta, t)$ \\
$\frac{1}{2} k x^{2}=\frac{1}{2} m v^{2}+m g(L+x) \sin \theta$ & $2:(R, t)$ & $2:(v, t)$ & $3:(v, \theta, t)$ \\
$R=v \cos \theta t$ & $2:(y, t)$ & $2:(v, t)$ & $1:(y)$ \\
$y=v \sin \theta t-\frac{1}{2} \mathrm{~g} t^{2}$ & $2:(y, R)$ & $1:(y)$ & Quadratic with trigonometry \\
$\tan \phi=\frac{-y}{R}$ & Nonquadratic & Quadratic & embedded \\
Type of equations & & & \\
\hline \hline
\end{tabular}

See Appendix A for the solutions. 
increasing closeness to the final correct solution, namely, identification, follow-up, and application of pertinent concepts. See Appendix B for the detailed coding scheme. The coding of the students' written solutions was done independently by two researchers and an inter-coder agreement of $86 \%$ was obtained. After discussion, the differences were resolved.

For concepts identification we coded the concepts identified by the students for tackling the sequential problems, with a particular interest in their identification of the two pertinent ones. For concepts follow-up, we looked at whether or not the students made a commitment to proceed with the identified pertinent concepts to solve the problems. For concepts application, we considered whether or not the students correctly used the concepts. For energy conservation, we specifically focused on the following:

- equation generated to relate the types of energy in the initial and final conditions,

- expression for height in the gravitational potential energy term when the block is at the crest of the ramp.

On application of projectile motion, we accounted for

- separating the block's velocity at the crest of the ramp into $x$ and $y$ components,

- the initial and final positions of the horizontal and vertical motion in the equation,

- using the angle $\phi$ to link the horizontal and vertical displacements traveled by the block.

We also scored the students' overall performance on the sequential synthesis problems. The total marks for the sequential tasks is 19 . The detailed marking scheme is in Appendix C. The grading was done independently by two researchers and an interreliability rate of $87 \%$ was obtained. Any difference in coding was discussed and resolved.

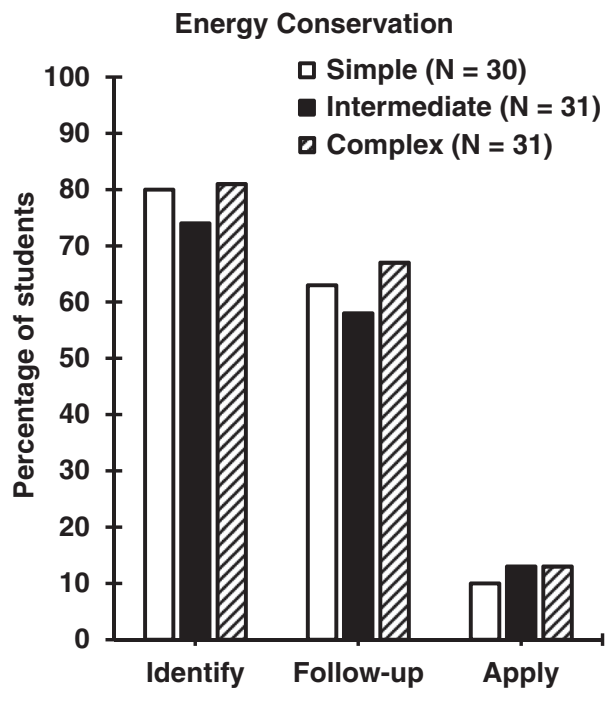

(a)
Individual interviews. - The individual interviews allowed us to gather in-depth information about students' actions when handling the sequential problems. In this study, the interviews were analyzed thematically for the following three main themes of interest: (i) factors cueing the identification of concepts, (ii) reasons for not identifying the appropriate concepts, and (iii) students' approaches to handle the sequential tasks, i.e., if they separate the situation presented in the task into segments or not. The interview protocol is in Appendix D.

\section{B. Results}

The sequential problems consist of two consecutive events, the spring launching the block and the block undergoing a trajectory. We present the findings on the effects of mathematical complexity on students' conceptual performance on each event followed by their overall score. We then report the students' approaches to handle the sequential synthesis tasks that were found in the interviews. Finally, we report the factors hinting students to use the pertinent concepts, and the reasons for their nonidentification.

\section{Written results}

Conceptual performance includes three levels: identification, follow-up, and correct application of the pertinent concepts. A $\chi^{2}$ test was performed at each level to detect any differences across the three versions of sequential synthesis problem. Figure 4 presents the percentages of students who correctly identified the pertinent concepts, followed-up on their use, and correctly applied them for the spring and the trajectory event, with respect to the task's mathematical complexity.

For energy conservation, Fig. 4(a), there was no significant difference between the three conditions at any analysis

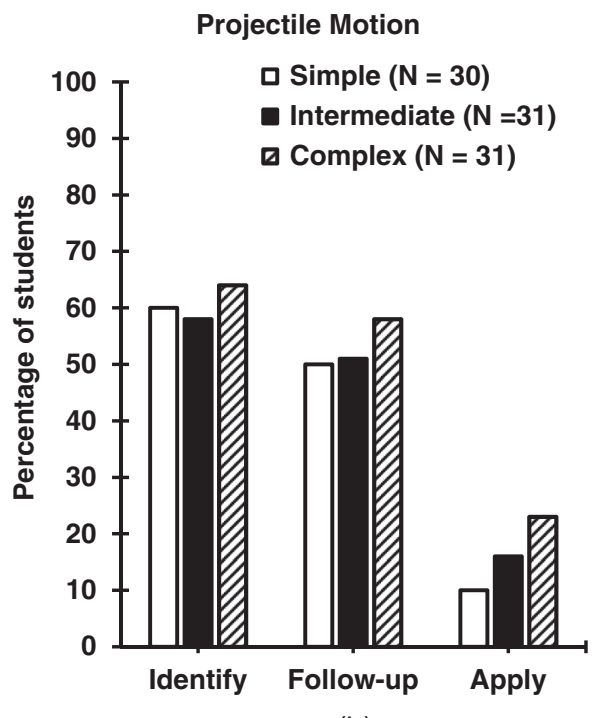

(b)

FIG. 4. Conceptual performance across sequential synthesis tasks with varying mathematical complexity for the (a) spring event and (b) trajectory event. 


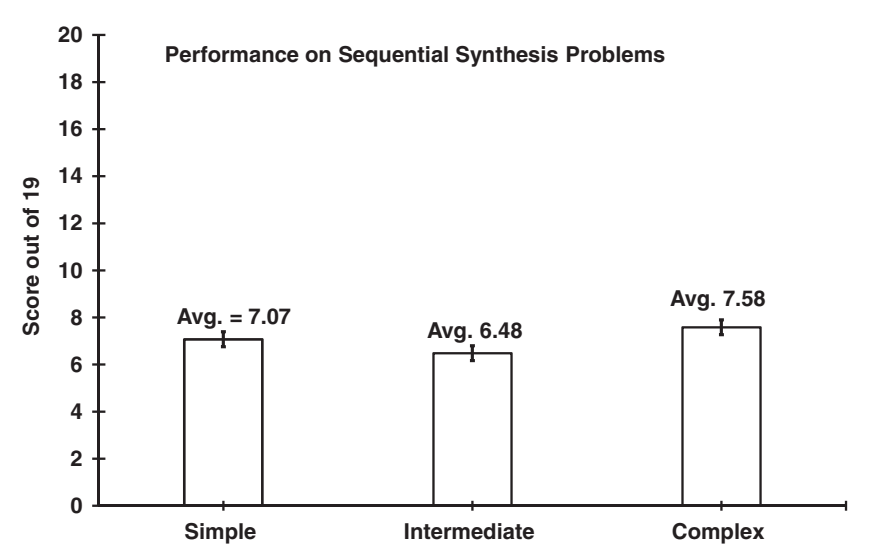

FIG. 5. Overall performance on the three sequential synthesis tasks. Error bars represent standard errors.

level (identification: $\left[\chi^{2}=0.458, p=0.795\right]$; follow-up: $\left[\chi^{2}=0.625, p=0.732\right]$; application: $\left[\chi^{2}=0.162, p=\right.$ 0.922]). Similarly, for projectile motion, Fig. 4(b), there was no significant difference between the three conditions at any analysis level (identification: $\left[\chi^{2}=0.285, p=\right.$ 0.867]; follow-up: [ $\chi^{2}=0.449, p=0.799$ ]; application: $\left.\left[\chi^{2}=1.77, p=0.413\right]\right)$. Thus, mathematical complexity did not appear to impact students' conceptual performance on the sequential synthesis problems.

For energy conservation, Fig. 4(a), the main mistakes students made when applying this pertinent concept are (i) omitting certain types of energy (the block's gravitational potential energy or kinetic energy when it is at the crest of the ramp), and (ii) formulating an incorrect height expression for the block's gravitational potential energy by disregarding the spring compression. These mistakes resulted in low performance on applying energy conservation. For projectile motion, Fig. 4(b), the students used incorrect expressions for the initial and/or final positions in the equations for the horizontal and vertical motion. They also incorrectly used trigonometry to relate the angle $\phi$ to the magnitude and direction of the horizontal and vertical displacements traveled by the block.
We scored the students' overall performance on the sequential synthesis tasks. The outcomes are presented in Fig. 5. The students' average score was nearly the same across the three versions of the problem $[F(2,91)=0.320$, $p=0.727]$, further indicating that mathematical complexity did not seem to affect the students' performance on the sequential synthesis tasks.

\section{Interview results}

We also explored if the students treated the situation presented in the sequential task in chunks or as a single event. We found that the students attended to the situation in two or multiple parts, and used two concepts for problem solving regardless of the task's mathematical complexity.

All the students who participated in the individual interviews used two concepts (either correct or incorrect) for problem solving. Based on their verbal and written responses, we found that they split the task into segments. From the verbal responses, we referred to the expressions they used to highlight their approaches to tackle the sequential tasks. They often employed the following expressions: (i) see it as two problems, the first part or problem and the second part or problem, (ii) spring problem and projectile problem, and (iii) split the problem into different parts or break down the problem into two separate tasks. Typical responses for students who completed the simple, intermediate and complex task are shown in Fig. 6.

Apart from the students' verbal expressions, we considered their written solutions for concrete indications of how they handled the situation in the sequential tasks. We noted that they drew vertical and/or horizontal lines on the given diagram as illustrated by Fig. 7. These actions indicate that the students viewed the problem as comprising either two parts (when one line is drawn from the crest of the ramp to the horizontal plane) or multiple parts (typically from the base of the ramp to the block's location, from the block to the crest of the ramp, and from the crest of the ramp to the landing spot).

Overall, the students divided the sequential synthesis problems into segments and used two concepts for problem solving irrespective of the task's mathematical complexity.

Student RP (simple task): "I saw it as two different problems. When I saw the second part I knew I had to use constant acceleration equations because it is a projectile motion. I know that the first part of the problem, the spring, will still be affecting the block."

Student HM (intermediate task): "I split the situation into different parts, like different sections, what happened here [shows block before its release], then what happen to this segment [shows section between the block and crest of the ramp] and then what happens as it is flying to this side [shows section between crest of the ramp to landing spot]."

Student AM (complex task): "We have the spring problem where we use energy. You need to find the value for energy, that's one whole chunk of math and then you do another whole different chunk of math for a different physics, the projectile problem, to find different values."

FIG. 6. Typical interview responses from the three versions of the sequential synthesis task. 


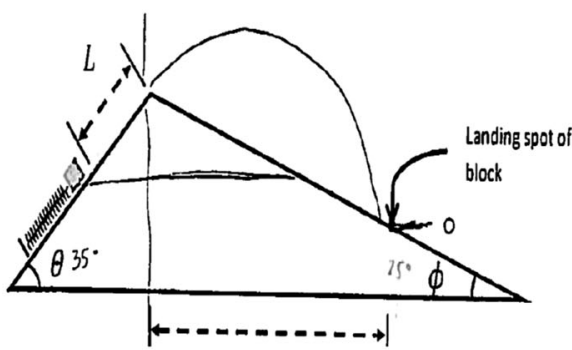

(a)

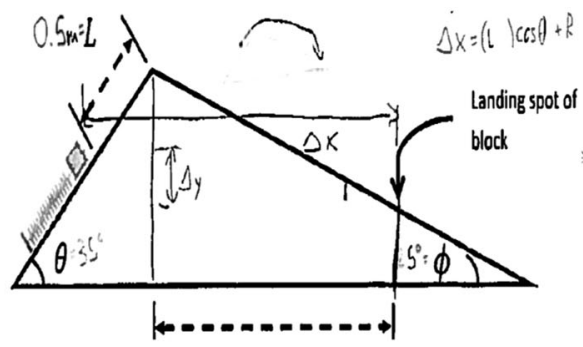

(b)

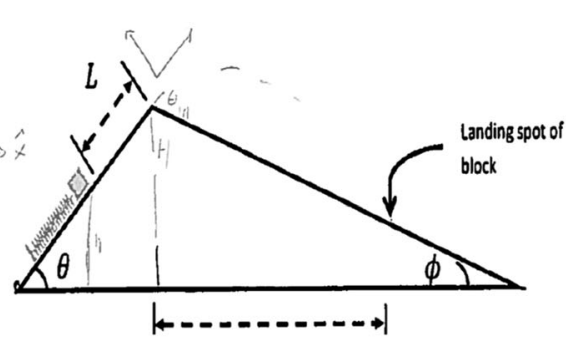

(c)

FIG. 7. Typical students' physical actions to sequential tasks for the (a) simple, (b) intermediate, and (c) complex version.

Individual interviews were also conducted to better understand the factors for why students used particular concepts. The prominent outcome was that the majority of the students identified the pertinent concepts, energy conservation, and projectile motion. The main factors prompting energy conservation were the ease of using this concept to tackle the problem, the task's surface features (the spring and the change in heights), and students' familiarity with related single-concept problems. Projectile motion was cued by the trajectory of the block. Typical interview responses for each factor are presented in Fig. 8.

Thus, the factors prompting the pertinent concepts for the sequential synthesis tasks were surface features (spring, change in heights, and object's trajectory), the ease of using the concept for solving the problem, and familiarity with related single-concept problems.

\section{STUDY 2: EFFECTS OF MATHEMATICAL COMPLEXITY ON STUDENT CONCEPTUAL PERFORMANCE IN SIMULTANEOUS SYNTHESIS PROBLEMS}

Study 2 explores students' conceptual performance when handling a simultaneous synthesis problem with varying mathematical complexity. Simultaneous synthesis problems (as shown in Fig. 9) involve a concurrent integration of multiple events and, hence, require the concurrent application of multiple fundamental principles.

\section{A. Method}

\section{Sample}

Study 2 was implemented during the Spring of 2015. The simultaneous problems were administered to 74 students enrolled in a second year mechanics course for physics majors. Of these 74 students, 66 signed up to complete the written tasks only. The remaining 8 students participated in individual interviews. Before participating in the study, all the students had learned the relevant concepts in their mechanics classes, where they had opportunities to handle multiple concept problems. The classes were traditionally taught and included a separate recitation that focused on students' practicing problem solving in groups.

\section{Simultaneous synthesis problems}

The simultaneous problems used in the study are depicted in Fig. 9. Similar to Study 1, the final set of problems was vetted with a group of physicists, physics education researchers, and students to ensure that the problem statements were clear, the quantitative information was rational, and the topics involved had been covered in class.

Two versions of the same problem were designed with different mathematical complexity levels, which we refer to

Student NY: "It is easier to use energy conservation. I thought about forces but it is going to be a nightmare to use Newtonian mechanics for the first part of the problem."

Student RV: "I recognise that there are changes in potential energy due to changes in heights and the easiest way to resolve changes in its spring energy is to use energy conservation."

Student SB: "I recognise energy conservation almost immediately because I saw the spring and we would need to find velocity in order to solve for $x$, spring compression."

Student NC: "Just experience with solving problems on energy conservation."

Student AW: "It didn't really occur to me to use anything else. I saw that it is a problem involving projectile, I wrote down these 2 things [show the equations for horizontal and vertical motion]. It has some mass which is going off in a trajectory."

FIG. 8. Typical student responses for factors cueing energy conservation and projectile motion. 


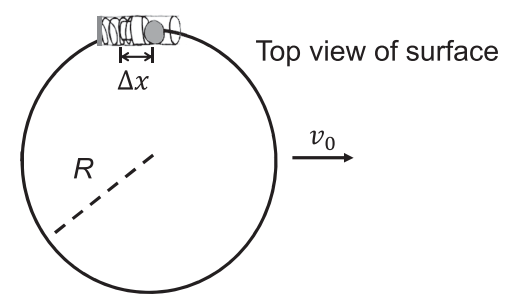

Simple: A disc of mass $3.0 \mathrm{~kg}$ and radius $R=0.75 \mathrm{~m}$ is sliding (face down) at velocity $v_{0}$ along a frictionless surface. A spring launcher is fixed outside the edge of the disc. The spring (spring constant, $\mathrm{k}=49.0 \mathrm{kN} / \mathrm{m}$ ) is compressed a distance $\Delta x$ and releases a projectile of mass $6.0 \mathrm{~kg}$ from its equilibrium position. After release, the velocity of the projectile relative to the surface is $v_{p}=11.0 \mathrm{~m} / \mathrm{s}$. Assume that the mass of the launcher is small and that the spring releases the projectile very rapidly. If the initial velocity of the disc was $v_{0}=5.0 \mathrm{~m} / \mathrm{s}$, what was the initial compression of the spring, $\Delta x$ ?

(a)

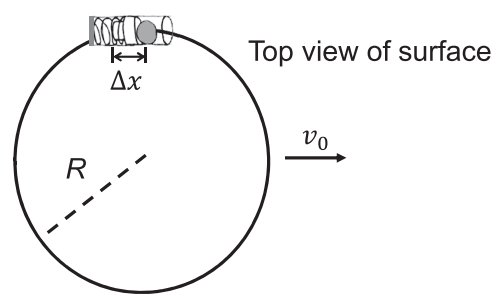

Complex: A disc of mass $3.0 \mathrm{~kg}$ and radius $R=0.75 \mathrm{~m}$ is sliding (face down) at velocity $v_{0}$ along a frictionless surface. A spring launcher is fixed outside the edge of the disc. The spring (spring constant, $\mathrm{k}=49.0 \mathrm{kN} / \mathrm{m}$ ) is compressed a distance $\Delta x$ and releases a projectile of mass $6.0 \mathrm{~kg}$ from its equilibrium position. After release, the velocity of the projectile relative to the surface is $v_{p}=11.0 \mathrm{~m} / \mathrm{s}$. Assume that the mass of the launcher is small and that the spring releases the projectile very rapidly. If the initial compression of the spring was $\Delta x=17.5 \mathrm{~cm}$, what was the initial velocity of the disc $v_{o}$ ?

(b)

FIG. 9. The two versions of the simultaneous synthesis problem.

as simple and complex. They both portray a disc sliding on a frictionless surface while a projectile being released by a spring launcher. When the compressed spring releases the projectile, the disc spins resulting in multiple concurrent events, including energy transfer and a change in translational and rotational motion of the system. Energy conservation, linear momentum conservation, and angular momentum around the center of the disc are the three pertinent concepts for tackling both versions of the problem.

Three fundamental equations, presented in Fig. 10, are needed to solve both tasks.

The application of energy conservation, linear momentum conservation, and angular momentum about the center of the disc results in the formulation of three equations. Specifically, the energy conservation equation, Eq. (1), connects the initial energy of the system (spring potential energy, and kinetic energy of the disc, and the projectile) with the final energy of the system (kinetic energy of the projectile and the disc, and the rotational kinetic energy of the disc). Equation (2), the linear momentum equation, relates the initial and final momentum of the system. The angular momentum equation, Eq. (3), relates the initial angular momentum of the system to its final angular momentum.

Information about the known and unknown variables and the types of equations to be manipulated for the two versions of the simultaneous synthesis task is shown in Table II.

\section{Administration of simultaneous synthesis problems}

The sample comprises 74 students. 66 of them signed up to complete the written tasks only. Each student was randomly assigned to one version of the task $(n=33$ for simple task; $n=33$ for complex task). There was no significant between-group difference in the average course grade $[F(1,65)=0.242, p=0.625]$, indicating that the students in the two groups were at the same level. The students completed the given task individually, without consulting their peers, as part of the "flexible homework" assignment for course credits [39]. Irrespective of the task's mathematical complexity, all students took approximately 30 minutes to solve the problems.

The remaining 8 students in the sample signed up to participate in a 1 hour individual interview session. Each student was randomly assigned to one version of the problem, simple $(n=4)$ and complex $(n=4)$. In each session, the student was initially allocated 30 minutes to solve the problem. The interview immediately followed, lasting a maximum of approximately 30 minutes.

\section{Analysis of simultaneous synthesis problems}

Written tasks.-Similarly to Study 1, the students' written solutions were analyzed in three levels at the order of increasing closeness to the final correct solution, namely,

$$
\begin{aligned}
& \text { Energy conservation: } \frac{1}{2} \mathrm{k} \Delta x^{2}+\frac{1}{2}(\mathrm{M}+\mathrm{m}) v_{o}^{2}=\frac{1}{2} \mathrm{~m} v_{p}^{2}+\frac{1}{2} \mathrm{M} v_{f}^{2}+\frac{1}{2} \mathrm{I} \omega^{2} \ldots \ldots \ldots(1) \\
& \text { Linear momentum conservation: }(\mathrm{M}+\mathrm{m}) v_{o}=\mathrm{M} v_{f}+\mathrm{m} v_{p} \ldots \ldots \ldots \ldots \ldots \ldots(2)
\end{aligned}
$$

Angular momentum conservation: $\mathrm{m} v_{o} R=\mathrm{m} v_{p} R+\mathrm{I} \omega$

FIG. 10. The three essential equations for solving the simultaneous synthesis problems. 
TABLE II. Known and unknown variables and types of equations across simultaneous synthesis tasks with increasing mathematical complexity ( $M$ : mass of disc, $R$ : radius of disc, $v_{o}$ : initial velocity of disc, $v_{f}$ : final velocity of disc, $\omega$ : angular velocity of disc, $m$ : mass of projectile, $v_{p}$ : final velocity of projectile, $k$ : spring constant, $\Delta x$ : initial compression of spring).

\begin{tabular}{lcc}
\hline \hline & Simple task & Complex task \\
\hline Common given information & & $M, m, R, k$, and $v_{p}$ \\
Additional given information & $v_{o}$ & $\Delta x$ \\
Variable to solve for & $\Delta x$ & $v_{o}$ \\
Number of unknowns in each equation & $1:(\Delta x)$ & $3:\left(v_{o}, v_{f}, \omega\right)$ \\
$\frac{1}{2} k \Delta x^{2}+\frac{1}{2}(M+m) v_{o}^{2}=\frac{1}{2} m v_{p}^{2}+\frac{1}{2} M v_{f}^{2}+\frac{1}{2} I \omega^{2}$ & $1:\left(v_{f}\right)$ & $2:\left(v_{o}, v_{f}\right)$ \\
$(M+m) v_{o}=M v_{f}+m v_{p}$ & $1:(\omega)$ & $2:\left(v_{o}, \omega\right)$ \\
$m v_{o} R=m v_{p} R+I \omega$ & Independent quadratic & Quadratic equation embedded \\
Types of equations & equation & in a system of 2 equations. \\
\hline \hline
\end{tabular}

See Appendix E for the solutions.

identification, follow-up, and application of pertinent concepts. See Appendix F for the detailed coding scheme. The coding of the students' written solutions was done independently by two researchers and an interreliability rate of $84 \%$ was obtained. Any difference in coding was discussed and resolved.

For concepts identification, we focused on the concepts considered by the students when they solved the simultaneous problems, particularly their identification of the pertinent ones. For concepts follow-up, we coded whether or not the students committed to using the identified pertinent concepts for tackling the problems. For concepts application, we considered whether or not the students correctly applied the pertinent concepts. We coded the types of energy, linear and angular momentum identified for the given physical situation. We then specifically attended to the types of energy, linear and angular momentum depicted for the initial and final conditions in the formulated equations. We also considered the velocity variable used for the projectile and the disc for the initial and final conditions in the equations. It requires an understanding that before being released by the compressed spring, the projectile and the disc have the same velocity. Once released, the projectile and the disc have different velocities.

Similarly to Study 1, we also scored the students overall performance on the simultaneous synthesis problems. The total marks for the simultaneous synthesis tasks is 28. The detailed marking scheme is in Appendix G. The grading was done independently by two researchers with an interreliability rate of $86 \%$. After discussion, the differences were resolved.

Individual interviews.-As before, the individual interviews were analyzed thematically to explore (i) possible factors that prompted students to consider certain concepts, (ii) reasons for students' failure to identify the pertinent concepts, and (iii) if the students treated the situation presented in the simultaneous tasks into chunks or as a single event. The interview protocol is in Appendix D.

\section{B. Results}

We first present the outcomes on the effects of mathematical complexity on students' conceptual performance for each event followed by their overall score. We then report the students' approaches to handle the simultaneous tasks. We finally report the factors prompting the pertinent concepts and reasons for their nonidentification.

\section{Written results}

Conceptual performance includes three levels of analysis: identification, follow-up, and correct application of the pertinent concepts. A $\chi^{2}$ test was performed at each level to detect any differences between the two simultaneous synthesis problems. Fig. 11 depicts the outcomes.

For energy transfer, Fig. 11(a), no significant betweengroup difference was noted in the number of students who identified the correct concept of energy conservation and followed up on its use $\left[\chi^{2}=0.982, p=0.322\right]$. For the complex task, we observed a substantial decrease in the percentage of students (more than 70\%) from "concept follow-up" to "concept application." For the simple task, the drop was around $50 \%$, leading to a significant difference in concept application between the two groups $\left[\chi^{2}=9.07\right.$, $p=0.0026]$. At the level of concept application, we found that the main conceptual error the students in both groups made when they applied energy conservation was omitting the disc's rotational kinetic energy. This mistake was more prominent for the complex task as opposed to the simple one, with a significant between-group difference $\left[\chi^{2}=4.98\right.$, $p=0.026]$. They also made errors associated with linear kinetic energy, omitting either the initial or the final kinetic energy of one of the objects in the system (i.e., disc or projectile). This mistake was more prominent for the 


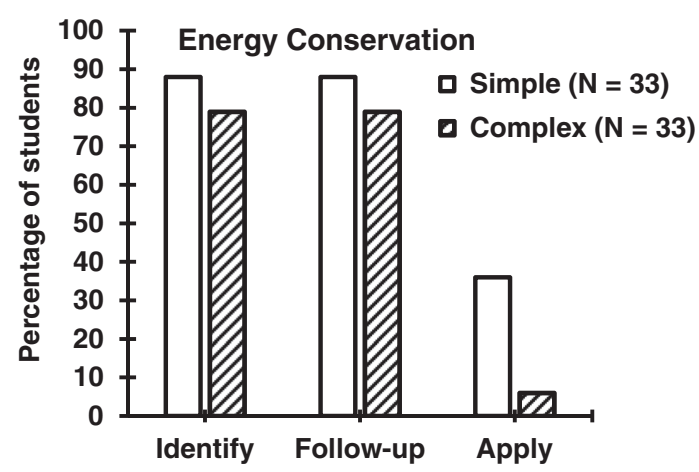

(a)

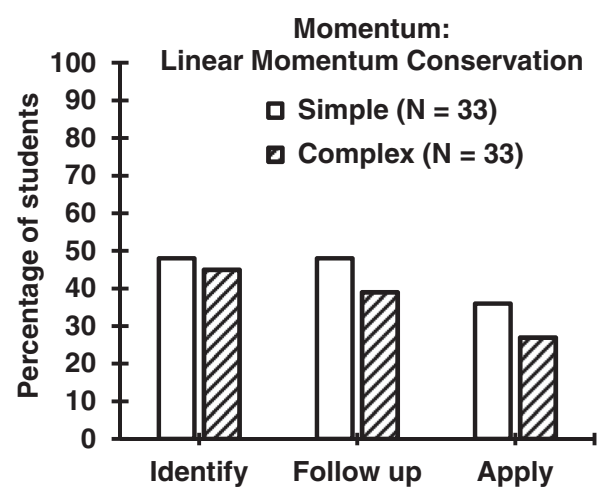

(b)

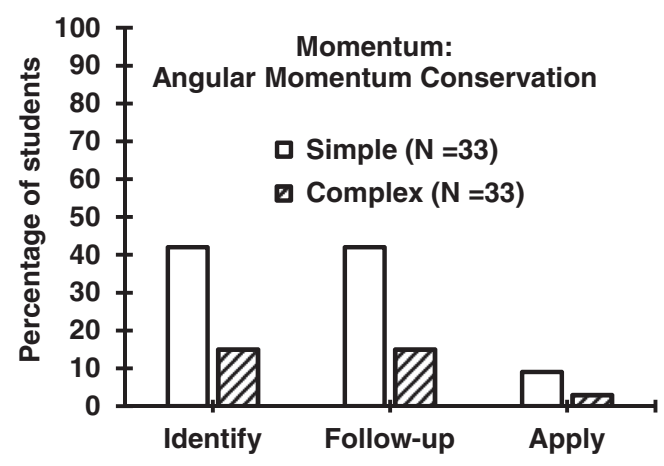

(c)

FIG. 11. Conceptual performance on simple and complex simultaneous synthesis tasks for (a) energy transfer, (b) translational motion, and (c) rotational motion.

complex task compared to the simple task, with a significant between-group difference $\left[\chi^{2}=12.83, p<0.001\right]$. This means that mathematical complexity influenced the students' correct application of energy conservation.

For translational motion, Fig. 11(b), there was no significant between-group difference for identifying linear momentum conservation $\left[\chi^{2}=0.061, p=0.805\right]$, following up on its use $\left[\chi^{2}=0.554, p=0.457\right]$, and correctly applying this pertinent concept $\left[\chi^{2}=0.629, p=0.428\right]$. Thus, mathematical complexity did not have an effect on the students' identification, follow-up, and correct application of linear momentum conservation.

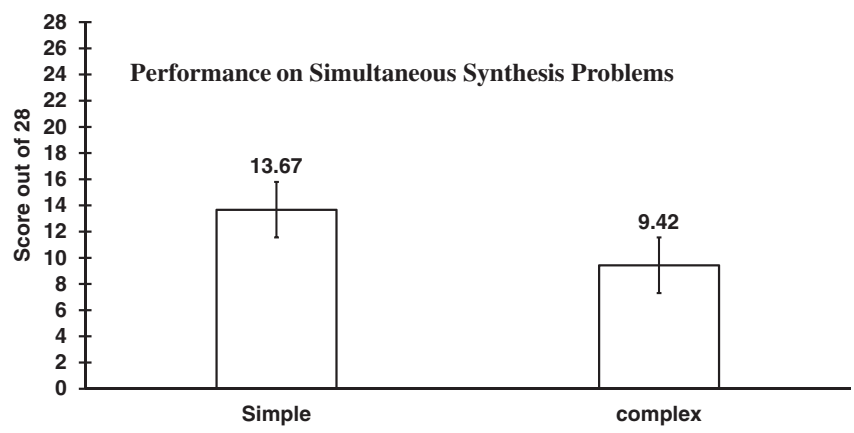

FIG. 12. Overall performance on the two simultaneous synthesis tasks. Error bars represent standard errors.
For rotational motion, Fig. 11(c), there was a significant between-group difference for identifying angular momentum conservation and following up on its use $\left[\chi^{2}=5.99\right.$, $p=0.014]$. There was no significant between-group difference in the number of students who correctly applied angular momentum conservation $\left[\chi^{2}=1.06, p=0.302\right]$. This indicates that mathematical complexity influenced the students' identification and commitment to using angular momentum conservation.

Overall, as the task's mathematical complexity increases, the students' conceptual performance on the simultaneous tasks decreases, specifically at the levels of concept identification and follow-up for angular momentum conservation, and concept application for energy conservation.

We scored the students' overall performance on the simultaneous synthesis tasks. The outcomes are presented in Fig. 12. The students' average score differed significantly between the simple and complex simultaneous tasks $[F(1,65)=4.19, p=0.004]$. This further indicates that mathematical complexity affected the students' performance on the simultaneous synthesis tasks.

\section{Interview results}

We also explored how the students approached the simultaneous synthesis problems, for example, whether they divided the situation into segments or considered it as 
Student SS (complex task): "We have a system in some initial configuration where the spring is compressed and then we have some final configuration where the spring is released. I take it as a problem where you look at the kinetic energy of the projectile before and after, and then you look at where that energy came from, from the compression of the spring."

Student RJ (simple task): "The spring will have an effect on the disc like pushing the other way. Since it is a spring acting on the top of the disc it would cause it to rotate backwards after the projectile is released."

FIG. 13. Typical interview responses from the two versions of the simultaneous synthesis task.

a single event. We found that they treated the situation in the simultaneous problem as a single event and tended to use one concept for problem solving, particularly for the complex task.

The majority of the students - three out of four-who completed the complex simultaneous synthesis problem only used one concept for problem solving. Among those who tackled the simple version of the task, only one out of four students used a single concept. Note that we considered the number of concepts used regardless of its correctness. Further, we looked at the students' verbal expressions as indications of their approaches to tackling the simultaneous tasks. Students who used one concept for problem solving often used the following expressions: (i) a system with an initial and a final point or configuration, (ii) there is a starting and an ending point. These expressions indicate that the students treated the situation in the problem as a single event comprising a before and an after condition, typically before and after the spring releases the projectile. Those who considered more than one concept used the expressions (i) the spring or the release of projectile creates the torque, (ii) the spring has an effect on the disc, and (iii) spring, ball, and disc are connected. These indicate that the students realized there was more than one event and highlighted the interrelation among them. Typical responses for students who completed the complex and the simple task are shown in Fig. 13.
Besides the students' verbal responses, we looked at their written solutions for concrete indications of how they tackled the situation in the simultaneous problems. We found that none of them left marks on the given diagram like in Study 1 where the students divided the situation in the sequential tasks into segments.

Overall, the students tended to treat the situation in the simultaneous synthesis tasks as a single event and used one concept for problem solving, mainly for the complex task.

From the individual interviews, we also investigated the factors prompting students to identify the appropriate concepts, and the reasons for their nonidentification. Unlike Study 1 , the prominent outcome in this case was that the majority of the students failed to identify the pertinent concepts of linear and angular momentum conservation. The main reasons for their nonidentification were (i) poor familiarity with and low confidence in these two concepts, and (ii) inability to unpack the given situation and make predictions by linking the task with learned physics concepts. However, similarly to Study 1, all the students identified energy conservation, which was cued by the task's surface features (spring, spring constant, before and after conditions) and the ease of using this concept to solve the problem. Figure 14 presents typical interview responses for each factor.

Hence, particular pertinent concepts were cued by the task's surface features and the ease of using the concepts for problem solving. The main reasons for their

\begin{abstract}
Student IB: "I didn't do too well in those two topics [linear and angular momentum conservation]. I am most familiar with conservation of energy. Linear momentum, angular momentum, that's just too complicated for this situation. I was trying to avoid thinking of these topics, using them. It's mostly that I don't like them."
\end{abstract}

Student SD: "I wasn't exactly sure what the ball was going to do, if it was going to shoot straight out or if it was constrained to the disc and I really don't know how the spring will affect the disc. I assume that [the disc] is lying flat on the table which doesn't necessarily have to be the case. If that's not the case, then I have to take into account some gravity. Not sure.[...] when I looked at the problem, I saw two points that I can use as a final and initial. When the spring is compressed versus when it is at equilibrium. I saw I can use energy conservation pretty simply to solve the problem."

Student JS: "I was given the spring constant. I knew that I can set up the equation for energy in the spring and then we are given the information about the velocity before and afterwards."

Student JD: "It just seems the obvious way to go about it. I usually like to start with energy conservation because I find that the easiest approach [...]. It makes the algebra a lot more simpler."

FIG. 14. Typical student responses for identifying and not identifying the pertinent concepts for the simultaneous synthesis problem. 
nonidentification were the students' lack of confidence in or familiarity with certain relevant concepts, and inability to unpack the given situation and make predictions by linking the task with learned physics concepts.

\section{CONCLUSIONS AND DISCUSSIONS}

There are two main observations from this study. First, we found that mathematical complexity did not influence students' conceptual performance on the sequential synthesis problems but had a noticeable, negative influence on their conceptual performance on the simultaneous synthesis problems. Second, analysis of interviews revealed that students tended to productively divide sequential synthesis problems into subtasks, and this division followed a physically valid chronological decomposition of the problem. In contrast, students did not tend to productively (or correctly) decompose simultaneous synthesis problems into components.

A possible confounding factor between the sequential (Study 1) and simultaneous (Study 2) synthesis problems used in this investigation is that they require different concepts. Therefore, the explanations for the differences in the influence of mathematical complexity between the two studies might include effects of the differences in physics concepts used as well as the problem type. That said, the concept of energy conservation appeared in both studies, where students' performances varied with mathematical complexity in the simultaneous case (Study 2) but showed no difference in the sequential case (Study 1). To a certain extent, this result mitigated concerns regarding such a possible confounding issue.

The sequential tasks are based on energy conservation and projectile motion. A relatively high percentage of students (60\%-80\%) identified these concepts regardless of the task's mathematical complexity. From individual interviews, the main reasons students provided for identifying the pertinent concepts were familiarity with similar single-concept problems, the ease of using the concepts for problem solving, and the task's surface features. Thus concept identification did not seem to be a bottleneck in solving sequential synthesis tasks with familiar concepts, and apparently the added mathematical complexity did not change this.

In contrast, the simultaneous problems had both high and low levels of concept identification depending on the concept. Consistent with Study 1, the concepts that had medium (around 50\% for linear momentum conservation) to high (around $88 \%$ for energy conservation) levels of identification were unaffected by mathematical complexity. However, the concept with low identification level (15\%$40 \%$ for angular momentum conservation) was significantly influenced by mathematical complexity. From individual interviews, the main reasons gathered for not identifying angular momentum conservation were students' less familiarity with or low confidence in the concept, and inability to unpack the given situation and to make predictions by linking the task with the learned physics concept. Hence, concept identification was a bottleneck to solving this simultaneous problem with an unfamiliar concept. This bottleneck was exacerbated when another layer of difficulty, mathematical complexity, was added to the problem.

In terms of concept application, for the sequential synthesis tasks, only a very small percentage of students correctly applied the concepts (10\%-25\%) for all conditions. The simplest explanation for the lack of influence of mathematical complexity may be that all students were "close to floor." Here, concept application appears to be a significantly large bottleneck which dominated other factors.

In contrast, for the simultaneous synthesis tasks, concept application did show some variation with respect to mathematical complexity, depending on the concept. Specifically, for energy conservation, the concept application success decreased significantly when mathematical complexity increased. As mentioned in the results section, the omission of rotational kinetic energy was predominantly responsible for this finding. The omission of rotational kinetic energy in the solutions might be explained by our observations of student interviews, which indicated that students had less familiarity with or low confidence in the concept. Further, the comparatively lower level of correct application of energy conservation in the high complexity condition could be explained in terms of cognitive load [40]. Specifically, as the task's mathematical complexity increases, the students had to handle more (and increasingly complicated) equations concurrently. This added difficulty may have interfered with the students' decisions regarding the application of energy conservation.

Another possible explanation for the difference in students' conceptual performance is the characteristics of the sequential and the simultaneous synthesis tasks. Regardless of the task's mathematical complexity, the students split the situation in the sequential synthesis task into segments and hence used more than one concept for problem solving. In contrast, the students treated the situation in the simultaneous synthesis task as a single event and used one concept, mainly for the complex task.

Thus, the characteristics of the synthesis task may have influenced the students' approach to tackle the given situation, and hence their conceptual performance. For the sequential tasks, even if the events are connected, it is possible to consider one event at a time. This may have prompted the students to attend to and unpack one event at a time, which facilitated their sense making of the individual events, the link between them, and hence the whole situation with its associated information. As such, the influence of mathematical complexity on students' conceptual performance in the sequential tasks may be bypassed. In contrast, for the simultaneous tasks, the events are concurrent such that it is necessary to consider all at once. The concurrence of the events may have made it difficult for the students to unpack the situation and prevented them from fully understanding the link among 
the different events. Hence, the simultaneous synthesis problem was mistakenly reduced by many students to a single event task with the application of one concept for problem solving. The inclusion of other components such as mathematical complexity and concepts which students were less familiar with or had low confidence in may have worsened the situation thus negatively affecting their conceptual performance on the simultaneous problems.

These results provide useful implications for teaching. From a pedagogical perspective, synthesis physics problems may promote problem solving as a learning tool and as an assessment tool. Synthesis problems combine two or more concepts to create a novel situation and emphasize qualitative task analysis [2]. Furthermore, synthesis problems cannot be solved by simple plug-and-chug. They require a blending of equations generated from applying multiple concepts. Besides understanding the concepts underlying the equations, students need to have a good sense of how the different mathematical expressions are connected. Overall, synthesis problems may support the development of an expertlike problem solving approach by channeling the students' focus on the underlying concepts and their application. They may also encourage students to attribute meaning to the mathematical tools, procedures, and symbols in the physics context.

\section{ACKNOWLEDGMENTS}

This work is supported by the National Science Foundation (Grant No. DRL - 1252399).

\section{APPENDIX A: TYPICAL SOLUTIONS FOR SEQUENTIAL SYNTHESIS PROBLEMS}

For the three sequential problems, we need the four equations:

$$
\begin{aligned}
& \text { Energy conservation: } \frac{1}{2} k x^{2}=\frac{1}{2} m v^{2}+m g(L+x) \sin \theta \\
& \text { Projectile motion: } R=v \cos \theta t \\
& y=v \sin \theta t-\frac{1}{2} g t^{2} \\
& \tan \phi=\frac{-y}{R}
\end{aligned}
$$

\begin{tabular}{|c|c|c|}
\hline Simple task & Intermediate task & Complex task \\
\hline From Eq. (A1): & From Eq. (A2): $t=\frac{R}{v \cos \theta}$ & From Eq. (A1): \\
\hline$\frac{m v^{2}}{2}=\frac{k x^{2}}{2}-\operatorname{mg}(L+x) \sin \theta$ & & $v^{2}=\frac{k x^{2}}{m}-2 g(L+x) \sin \theta$ \\
\hline$v=\left[\frac{k x^{2}}{m}-2 g(L+x) \sin \theta\right]^{\frac{1}{2}}$ & Plug Eq. (A4) and $t=\frac{R}{v \cos \theta}$ in Eq. (A3): & Plug values gives: \\
\hline Plug values gives $v=2.87 \mathrm{~m} / \mathrm{s}$. & $-R \tan \phi=v \sin \theta\left(\frac{R}{v \cos \theta}\right)-\frac{g R^{2}}{2 v^{2} \cos ^{2} \theta}$ & $v^{2}=15-11.76 \sin \theta \mathrm{m} / \mathrm{s}$ \\
\hline Plug Eqs. (A2) and (A4) in Eq. (A3): & Simplify and isolate for $v$ & From Eq. (A2): $t=\frac{R}{v \cos \theta}$ \\
\hline$-v \cos \theta t \tan \phi=v \sin \theta t-\frac{g t^{2}}{2}$ & $v=\left[\frac{g R}{2 \cos ^{2} \theta(\tan \phi+\tan \theta)}\right]^{\frac{1}{2}}$ & $\begin{array}{c}\text { Plug Eq. (A4) and } t=\frac{R}{v \cos \theta} \text { in Eq. (A3) } \\
\text { and simplify }\end{array}$ \\
\hline Simplify and isolate for $t$ & Plug values gives $v=2.87 \mathrm{~m} / \mathrm{s}$ & $\tan \phi=\tan \theta-\frac{g R}{2 v^{2} \cos ^{2} \theta}$ \\
\hline$t=\frac{2 v(\cos \theta \tan \phi+\sin \theta)}{g}$ & $\begin{array}{l}\text { From Eq. (A1): plug } v=2.87 \mathrm{~m} / \mathrm{s} \text { and } \\
\text { other values and simplify: }\end{array}$ & $\begin{array}{l}\text { Plug values and expression for } v^{2} \text { and } \\
\text { simplify: }\end{array}$ \\
\hline Plug values gives $t=0.560 \mathrm{~s}$ & $\begin{array}{c}1500 x^{2}-11.24 x-13.74=0 \\
\text { Isolate for } x\end{array}$ & $\frac{12.74}{(30-23.52 \sin \theta) \cos ^{2} \theta}-\tan \theta=0.466$ \\
\hline From Eq. (A2), plug values gives: & $x=\frac{11.24 \pm \sqrt{(11.24)^{2}-4(1500)(-13.74)}}{2(1500)}$ & Use identities and isolate for $\theta$ : \\
\hline$R=(2.87) \cos 35(0.560)=1.3 \mathrm{~m}$ & $x=0.1 \mathrm{~m}$ & $\theta=35^{\circ}$ \\
\hline
\end{tabular}

\section{APPENDIX B: CODING SCHEME FOR SEQUENTIAL SYNTHESIS PROBLEMS}

\section{Level I: Identification of pertinent concepts}

Level I deals with identification of the pertinent concepts, i.e., energy conservation and projectile motion for the spring and trajectory event, respectively. As long as a student mentioned energy conservation and/or projectile motion, the student was considered to be in this category.

Level II: Follow-up on pertinent concepts

Level II is concerned with follow-up on the pertinent concepts. It refers to whether or not the students committed to proceed with energy conservation and projectile motion to solve the problems. Students who did use the relevant concepts 
are in this category. Those who later discarded the appropriate concept(s) to use other irrelevant one(s) are not considered in this category.

\section{Level III: Correct application of pertinent concepts}

Level III pertains to correct application of the pertinent concepts. For the spring event, level III is associated with the correct application of energy conservation. It focuses on whether the students (i) formulate an energy conservation equation with the relevant types of energy for the initial condition (spring potential energy) and the final condition (kinetic energy and gravitational potential energy of the block), i.e., $1 / 2 k x^{2}=1 / 2 m v^{2}+m g h$, (ii) use the appropriate height expression for the gravitational potential energy term i.e., formulate equation $1 / 2 k x^{2}=1 / 2 m v^{2}+m g(L+x) \sin \theta$. The students have to consider the block's equilibrium position $L$, and the spring compression $x$, when formulating the height expression.

For the trajectory event, level III is associated with the correct application of projectile motion: (i) separating the block's velocity into horizontal and vertical components, (ii) equation for horizontal motion, $R=v \cos \theta t$, (iii) trigonometry relating angle $\phi$, magnitude and direction of variables, $\tan \phi=-y / R$, and (iv) equation for vertical motion, $-y=-R \tan \phi=v \sin \theta t-1 / 2 g t^{2}$.

\section{APPENDIX C: MARKING SCHEME FOR SEQUENTIAL SYNTHESIS PROBLEMS}

\begin{tabular}{|c|c|}
\hline & Mark \\
\hline \multicolumn{2}{|l|}{ Identification of pertinent concepts } \\
\hline - Energy conservation & 1 \\
\hline - Projectile motion & 1 \\
\hline \multicolumn{2}{|l|}{ Application of pertinent concepts } \\
\hline \multicolumn{2}{|l|}{ Energy conservation } \\
\hline - Identify spring potential energy & 1 \\
\hline - Identify kinetic energy of block & 1 \\
\hline - Identify gravitational potential energy of block & 1 \\
\hline $\begin{array}{l}\text { - Correct equation identifying the relevant types of } \\
\text { energy for the initial and final conditions, i.e., } \\
1 / 2 k x^{2}=1 / 2 m v^{2}+m g h\end{array}$ & 1 \\
\hline $\begin{array}{l}\text { - Correct expression for height value in gravitational } \\
\text { potential energy term, i.e., } m g(L+x) \sin \theta\end{array}$ & 1 \\
\hline \multicolumn{2}{|l|}{ Projectile motion } \\
\hline $\begin{array}{l}\text { - Correct equation for horizontal motion, } \\
\text { i.e., } R=v \cos \theta t \text { or } x(t)=v \cos \theta t\end{array}$ & 1 \\
\hline $\begin{array}{l}\text { - Correct equation using the angle } \phi \text { to relate the } \\
\text { horizontal and vertical displacements, } \\
\text { i.e., } \tan \phi=-y / R\end{array}$ & \\
\hline $\begin{array}{l}\text {-Correct opposite }(y) \text { and adjacent }(R) \text { variables in the } \\
\text { equation }\end{array}$ & 1 \\
\hline -Correct direction & 1 \\
\hline $\begin{array}{l}\text { - Correct equation for vertical motion, i.e., } \\
-R \tan \phi=v \sin \theta t-1 / 2 g t^{2}\end{array}$ & \\
\hline -Initial position & 1 \\
\hline -Final position & 1 \\
\hline
\end{tabular}

(Table continued)
(Continued)

-Consistency in sign for direction of motion

-Velocity component

1

Equations

- Combine projectile motion equations and either isolate 2 or eliminate time

- Combine projectile motion equations but do not isolate 1 or eliminate time

- Combine energy conservation and projectile motion 2 equations to find the required variable

- Instead of actually combining the equations, the students 1 mention the steps to be implemented

Final value

- Correct answer 1

The coding scheme in Appendix B focuses only on the conceptual aspect of the problem (identification, follow-up, and application of pertinent concepts). The students' overall performance additionally includes their approaches to handling quantitative equations, which we examined in a similar manner that an instructor would grade students' work. Further, a key element of synthesis problem solving is the combination of equations, immediately following the application of multiple concepts. Thus the marking scheme here is designed to include this additional, important step, which is not fully captured in the conceptual aspect of the problem solving.

\section{APPENDIX D: INTERVIEW PROTOCOL FOR STUDY 1 AND 2}

- What concepts did you use?

- What lead you to use this concept/these concepts? Did you have any particular reason(s) for using this concept/these concepts?

- You said you use this concept/these concepts. Are there any other concept(s) which you think are applicable to this problem? Can you elaborate on your response?

- Apart from the concept(s) you mentioned, what else did you use or think you can use? Did it cross your mind that you can use this concept(s) to solve this problem? Can you tell me more about why you did not think about this concept/these concepts?

- Tell me, in your own words, what the task is about. Guide me over your solution. How did you proceed to solve this problem?

- When you were given this task, how did you think about it before you started solving it?

- You said that you use two different methods/you mentioned that you attempted the problem in stages. Can you explain what you mean by that? Can you elaborate on your response?

- You mentioned that you used the best approach to tackle this problem. Can you tell me more about that? 


\section{APPENDIX E: TYPICAL SOLUTIONS FOR SIMULTANEOUS SYNTHESIS PROBLEMS}

For both versions of the simultaneous problem, we need the three equations:

$$
\begin{aligned}
& \text { Energy conservation: } \frac{1}{2} k \Delta x^{2}+\frac{1}{2}(M+m) v_{o}^{2}=\frac{1}{2} m v_{p}^{2}+\frac{1}{2} M v_{f}^{2}+\frac{1}{2} I \omega^{2} \\
& \text { Linear momentum conservation: }(M+m) v_{o}=M v_{f}+m v_{p} \\
& \text { Angular momentum conservation: } m v_{o} R=m v_{p} R+I \omega
\end{aligned}
$$

\begin{tabular}{cc}
\hline \hline Simple task & Intermediate task \\
\hline
\end{tabular}

From Eq. (E2):

$v_{f}=\frac{(M+m) v_{o}-m v_{p}}{M}$

Plug values gives $v_{f}=-7 \mathrm{~m} / \mathrm{s}$

From Eq. (E3):

$\omega=\frac{m R v_{o}-m R v_{p}}{\frac{1}{2} m R^{2}}$

Plug values gives $\omega=-32 \mathrm{rad} / \mathrm{s}$

From Eq. (E1), simplify and isolate for $\Delta x$ :

$\Delta x= \pm \sqrt{\frac{m v_{p}^{2}+M v_{f}^{2}+\frac{1}{2} M R^{2} \omega^{2}-(M+m) v_{o}^{2}}{k}}$

Plug values gives $\Delta x= \pm 0.176 \mathrm{~m}, \Delta x=17.6 \mathrm{~cm}$.
From Eq. (E2):

$v_{f}=\frac{(m+M) v_{o}-m v_{p}}{M}$

From Eq. (E3):

$\omega=\frac{m v_{o} R-m v_{p} R}{I}=\frac{m R}{I}\left(v_{o}-v_{p}\right)$

Plug expressions for $v_{f}, \omega$, and $I=1 / 2 M R^{2}$ in Eq. (E1):

$\frac{1}{2} k \Delta x^{2}+\frac{1}{2}(M+m) v_{o}^{2}=\frac{1}{2} m v_{p}^{2}+\frac{1}{2} \frac{M}{m^{2}}\left[(M+m) v_{o}-m v_{p}\right]^{2}$ $+\frac{1}{2} \mathrm{I}\left(\frac{m R}{\mathrm{I}}\right)^{2}\left(v_{o}-v_{p}\right)^{2}$

Plug values, simplify, and isolate for $v_{o}$ :

$$
\begin{aligned}
& (-42) v_{o}^{2}+(924) v_{o}-3581.375=0 \\
& v_{o}=\frac{22 \pm \sqrt{(22)^{2}-4(85.27)}}{2} \\
& v_{o}=(5.0 \mathrm{~m} / \mathrm{s}, 17 \mathrm{~m} / \mathrm{s}), v_{o}=5.0 \mathrm{~m} / \mathrm{s} .
\end{aligned}
$$

\section{APPENDIX F: CODING SCHEME FOR SIMULTANEOUS SYNTHESIS PROBLEMS}

\section{Level I: Identification of pertinent concepts}

Level I deals with the identification of the pertinent concepts, i.e., energy conservation, linear, and angular momentum conservation for the event relating to energy transfer, translational motion, and rotational motion, respectively. As long as a student identified one of these pertinent concepts, the student is considered to be in this category.

\section{Level II: Follow-up on pertinent concepts}

Level II is associated with follow-up on the pertinent concepts. It refers to whether or not the student committed to proceed with energy conservation, linear and angular momentum conservation to solve the problems. Students who did commit to use these pertinent concepts are in this category. Those who later abandon the appropriate concept(s) in favor of irrelevant one(s) are not considered in this category.

Level III: Correct application of pertinent concepts

Level III is concerned with the correct application of the pertinent concepts. For energy transfer, level III identifies the correct types of energy in the given situation, i.e., spring potential energy, kinetic energy of the projectile and the disc, and the disc's rotational kinetic energy. It also considers if the students correctly identified the types of energy for the initial condition (spring potential energy and kinetic energy of the disc and projectile combined) and final conditions (kinetic energy of the projectile and disc, disc's rotational kinetic energy) in the energy conservation equation. Level III also considers the velocity variables for the initial and final conditions, i.e., $1 / 2 k \Delta x^{2}+1 / 2(M+m) v_{o}^{2}=1 / 2 M v_{f}^{2}+1 / 2 m v_{p}^{2}+1 / 2 I \omega^{2}$.

For translational motion, level III considers whether the students identified linear momentum due to both the projectile and the disc. It also focuses on whether the students correctly used linear momentum conservation to link the initial and final linear momentum due to the disc and the projectile. This level also considers the correct velocity variables used for the initial and final condition, i.e., $(M+m) v_{o}=M v_{f}+m v_{p}$.

For rotational motion, level III considers if the students considered the angular momentum due to both the projectile and the disc. It also emphasizes if the students correctly used angular momentum conservation to link the initial and final angular momentum due to the projectile, and the final 
angular momentum due to the disc. Further, this level focuses on the appropriate velocity variables for the initial and final condition, i.e., $m v_{o} R=m v_{p} R+I \omega$.

\section{APPENDIX G: MARKING SCHEME FOR SIMULTANEOUS SYNTHESIS PROBLEMS}

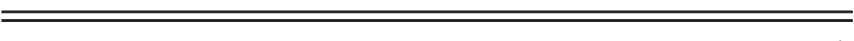

Identification of pertinent concepts

- Energy conservation

- Linear momentum conservation

- Angular momentum conservation

Application of pertinent concepts

Energy conservation

- Identify spring potential energy

- Identify kinetic energy of system, i.e., disc and projectile 1 combined

- Identify kinetic energy of projectile

- Identify kinetic energy of disc

- Identify rotational kinetic energy of disc

- Correct equation relating correct types of energy for initial 4 and final conditions and use of correct velocity variables, i.e., $\frac{1}{2} k \Delta x^{2}+\frac{1}{2}(M+m) v_{o}^{2}=\frac{1}{2} m v_{p}^{2}+\frac{1}{2} M v_{f}^{2}+\frac{1}{2} I \omega^{2}$

Linear momentum conservation

- Identify linear momentum due to system, i.e., disc and 1 projectile combined

(Table continued)
(Continued)

- Identify linear momentum due to projectile

- Identify linear momentum due to disc

- Correct equation relating correct types of linear momentum for initial and final conditions and use of correct velocity variables, i.e., $(M+m) v_{o}=M v_{f}+m v_{p}$

Angular momentum conservation

- Identify angular momentum due to projectile

- Identify angular momentum due to disc

Correct equation relating relevant types of angular momentum for initial and final conditions and use of correct velocity variables, i.e., $m v_{o} R=m v_{p} R+I \omega$

Equations

- Combine equations and isolate for required variable 3

- Combine equations but do not isolate for required variable 2

- Instead of actually combining the equations, the students 1 mention the steps to be implemented

Final value

- Correct answer

Similar to Study 1, the marking scheme for Study 2 was designed to additionally cater for the students' manipulation of equation. This was done since (i) we graded the students' overall performance similar to an instructor's, and (ii) a key aspect of the synthesis problem is the merging of equations, emerging on application of multiple concepts, for solving the final value.
[1] B. Ibrahim and N. S. Rebello, Representational task formats and problem solving strategies in kinematics and work, Phys. Rev. ST Phys. Educ. Res. 8, 010126 (2012).

[2] L. Ding, N. Reay, A. Lee, and L. Bao, Exploring the role of conceptual scaffolding in solving synthesis problems, Phys. Rev. ST Phys. Educ. Res. 7, 020109 (2011).

[3] L. Hsu, E. Brewe, T. M. Foster, and K. A. Harper, Resource Letter RPS-1: Research in problem solving, Am. J. Phys. 72, 1147 (2004).

[4] J. L. Docktor and J. P. Mestre, Synthesis of disciplinebased education research in physics, Phys. Rev. ST Phys. Educ. Res. 10, 020119 (2014).

[5] D. P. Maloney, Getting started in PER, Reviews in PER (American Association of Physics Teachers College Park, MD, 2011), Vol. 2.

[6] P. T. Hardiman, R. Dufresne, and J. P. Mestre, The relation between problem categorization and problem solving among experts and novices, Mem. Cogn. 17, 627 (1989).

[7] P. B. Kohl and N. D. Finkelstein, Patterns of multiple representation use by experts and novices during physics problem solving, Phys. Rev. ST Phys. Educ. Res. 4, 010111 (2008).

[8] P. Heller, R. Keith, and S. Anderson, Teaching problem solving through cooperative grouping. Part 1: Group versus individual problem solving, Am. J. Phys. 60, 627 (1992).

[9] P. B. Kohl and N. D. Finkelstein, Effect of representation on students solving physics problems: A fine-grained characterization, Phys. Rev. ST Phys. Educ. Res. 2, 010106 (2006).

[10] D. E. Meltzer, Relation between students' problem solving performance and representational format, Am. J. Phys. 73, 463 (2005).

[11] B. Ibrahim and N. S. Rebello, Role of mental representations in problem solving: Students' approaches to nondirected tasks, Phys. Rev. ST Phys. Educ. Res. 9, 020106 (2013).

[12] C. A. Ogilvie, Changes in students' problem-solving strategies in a course that includes context-rich, multifaceted problems, Phys. Rev. ST Phys. Educ. Res. 5, 020102 (2009).

[13] P. D. Antonenko, C. A. Ogilvie, D. S. Niderhauser, J. Jackman, P. Kumsaikaew, R. R. Marathe, and S. M. Ryan, Understanding student pathways in context-rich problems, Educ. Inf. Technol. 16, 323 (2011).

[14] R. Q. Xu, Ph.D. thesis, University of Minnesota 2013.

[15] S. Lin and C. Singh, Using isomorphic problems to learn introductory physics, Phys. Rev. ST Phys. Educ. Res. 7, 020104 (2011). 
[16] L. Ding, N. W. Reay, A. Lee, and L. Bao, Using Conceptual Scaffolding to Foster Effective Problem Solving, AIP Conf. Proc. 1179, 129 (2009).

[17] L. Ding, N. W. Reay, A. F. Heckler, and L. Bao, Sustained Effects of Solving Conceptually-scaffolded Synthesis Problems, AIP Conf. Proc. 1289, 133 (2010).

[18] D. R. White, R. Badeau, A. F. Heckler, and L. Ding, Bottlenecks in solving synthesis problems, in Proceedings of the Physics Education Conference, Minneapolis, MN, 2014, edited by P. V. Engelhardt, A. D. Churukian, and D. L. Jones (AIP, New York, 2014).

[19] K. R. Koedinger, M. W. Alibali, and M. J. Nathan, Trade-offs between grounded and abstract representations: evidence from algebra problem solving, Cogn. Sci. 32, 366 (2008).

[20] N. Heffernan and K. R. Koedinger, The composition effect in symbolizing: The role of symbol production vs text comprehension, in Proceedings of the Nineteenth Annual Conference of the Cognitive Science Society, edited by M. G. Shafto and P. Langley (Erlbaum, Hillsdale, NJ, 1997), pp. 307-312.

[21] J. Larkin, J. McDermott, D. P. Simon, and H. A. Simon, Expert and novice performance in solving physics problems, Science 208, 1335 (1980).

[22] A. Van Heuvelen, Overview, Case Study Physics, Am. J. Phys. 59, 898 (1991).

[23] D. P. Maloney, in Handbook of Research on Science Teaching and Learning Project, edited by D. Gabel (MacMillan, London, 1994).

[24] D. P. Simon and H. A. Simon, Individual differences in solving physics problems in Children's Thinking: What Develops?, edited by R. S. Siegler (Erlbaum, Hillsdale, N.J., 1978).

[25] F. Reif and J. Heller, Knowledge structure and problem solving in physics, Educ. Psychol. 17, 102 (1982).

[26] E. F. Redish, Problem solving and the use of math in physics course, in Proceedings of the World View on Physics Education in 2005: Focusing on Change, Delhi (2005), https://arxiv.org/ftp/physics/papers/0608/0608268.pdf.

[27] T. Bing and E. F. Redish, Analyzing problem solving using math in physics: Epistemological framing via warrants, Phys. Rev. ST Phys. Educ. Res. 5, 020108 (2009).
[28] R. E. Pepper, S. V. Chasteen, S. J. Pollock, and K. K. Perkins, Observations on student difficulties with mathematics in upper division electricity and magnetism, Phys. Rev. ST Phys. Educ. Res. 8, 010111 (2012).

[29] E. Kuo, M. M. Hull, A. Gupta, and A. Elby, How students blend conceptual and formal mathematical reasoning in solving physics problems, Sci. \& Educ. 97, 32 (2013).

[30] R. H. Wenger, Cognitive science and algebra learning in Cognitive Science and Mathematics Education, edited by A. H. Schoenfeld (Erlbaum, Hillsdale, N.J., 1987).

[31] K. R. Koedinger and M. J. Nathan, The real story behind story problems: effects of representations on quantitative reasoning, J. Learn. Sci. 13, 129 (2004).

[32] L. N. Walsh, R. G. Howard, and B. Bowe, Phenomenographic study of students' problem solving approaches in physics, Phys. Rev. ST Phys. Educ. Res. 3, 020108 (2007).

[33] M. T. H. Chi, P. Feltovich, and R. Glaser, Categorization and representation of physics problems by experts and novices, Cogn. Sci. 5, 121 (1981).

[34] J. Tuminaro and E. F. Redish, Elements of a cognitive model of physics problem solving: Epistemic games, Phys. Rev. ST Phys. Educ. Res. 3, 020101 (2007).

[35] B. L. Sherin, How students understand physics equations, Cognit. Instr. 19, 479 (2001).

[36] A. Van Heuvelen, Learning to think like a physicist: A review of research-based instructional strategies, Am. J. Phys. 59, 891 (1991).

[37] W. J. Leonard, R. J. Dufresne, and J. P. Mestre, Using qualitative problem-solving strategies to highlight the role of conceptual knowledge in solving problems, Am. J. Phys. 64, 1495 (1996).

[38] R. J. Dufresne, W. J. Gerace, and W. J. Leonard, Solving physics problems with multiple representations, Phys. Teach. 35, 270 (1997).

[39] A. F. Heckler and E. C. Sayre, What happens between pre-and post-tests: Multiple measurements of student understanding during an introductory physics course, Am. J. Phys. 78, 768 (2010).

[40] J. Sweller, Cognitive Load during Problem Solving: Effects on Learning, Cogn. Sci. 12, 257 (1988). 